\title{
Recovery of a Humanoid Robot from a Destabilising Impact.
}

\author{
Wallbridge, CD
}

http://hdl.handle.net/10026.1/10514

\section{TAROS}

Springer

All content in PEARL is protected by copyright law. Author manuscripts are made available in accordance with publisher policies. Please cite only the published version using the details provided on the item record or document. In the absence of an open licence (e.g. Creative Commons), permissions for further reuse of content should be sought from the publisher or author. 


\title{
Recovery of a Humanoid Robot from a Destabilising Impact
}

\author{
Christopher D. Wallbridge and Guido Bugmann \\ Plymouth University, Plymouth PL4 8AA, UK \\ Christopher.Wallbridge@plymouth.ac.uk G.Bugmann@plymouth.ac.uk
}

\begin{abstract}
This paper examines the case of a bipedal robot under an external impact along the axis of the two supporting feet. The dynamics of the robot is modelled using the 3-Mass Linear Inverted Pendulum Model. The model shows that, for impacts below a given threshold, the robot recovers naturally and no corrective action is required. For larger, destabilising impacts, this paper described how to calculate a single or a sequence of corrective steps. The key information used for the calculations is the initial velocity generated by the impact. The behaviour of the model for various initial configurations and impact parameters is illustrated by simulations.
\end{abstract}

Keywords: Humanoid, Robot, Dynamics, Impact, Corrective Stepping, Bipedal, LIPM

\section{Introduction}

The detection of destabilising events and the prevention of falls is a crucial functionality of future real-world walking robots. Notably, the IEEE International Conference on Robotics and Automation 2016 had a workshop devoted to Legged Robot Falling: Fall Detection, Damage Prevention, and Recovery Actions ${ }^{1}$. The work presented here focuses on bipedal Humanoid robots. These robots are unstable when compared to other robots with wheels, or additional legs. Falls can be costly, both in the time taken for the robot to recover and in potential repairs for any damage sustained. To allow humanoid robots to be deployed in a real world requires that they be able to take corrective action to unforeseen impacts.

\section{The 3-Mass Linear Inverted Pendulum Model}

Initial work on modelling gait used an Inverted Pendulum Model (IPM) with a single mass located on top of a massless pole [1]. The IPM has been further developed into a Linear Inverted Pendulum Model (LIPM) for use in robot controllers [3]. The LIPM adds a constraint into the motion of the pendulum,

\footnotetext{
${ }^{1}$ http://www.icra2016.org/
} 
keeping the height of the mass above the plane of motion at a constant distance. This simplifies computation by decoupling the foward and lateral motions.

While functional, this model proved to be insufficient when used in a robot controller. Work by Yuping et al [4] showed the importance of the mass distribution in a walking system. Therefore, an improvement to the model would also be modelling the mass distribution in the system.

Bugmann [2] expanded upon the LIPM to create a 3-Mass Linear Inverted Pendulum Model (3-MLIPM). By looking at torques created by gravity and how they relate to propulsion torques, the following formula can be found for a system of $n$ number of joints and masses:

$$
\sum_{i=1}^{n} m_{i} \ddot{x}_{i} z_{i}=\sum_{i=1}^{n} g m_{i} x_{i} \quad \sum_{i=1}^{n} m_{i} \ddot{y}_{i} z_{i}=\sum_{i=1}^{n} g m_{i} y_{i}
$$

This torque equation (1) can then be further used in an equation for the forward walking gait of the robot.

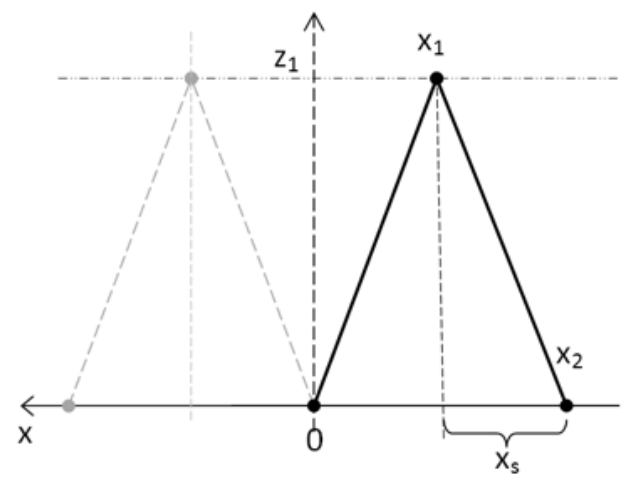

Fig. 1. 3-Mass robot model for a walking gait, with the motion going towards the left. The masses are linked together by virtual poles that maintain a constant height $\left(z_{1}\right)$. Note that in a real robot the height constance is achieved by using articulated knee joints. The masses $m_{1}$ and $m_{2}$ are at $\left(x_{1}, z_{1}\right)$ and $\left(x_{2}, 0\right)$ respectively. $m_{3}$ is located at $(0,0)$ and does not affect the dynamics of the system. [2]

Using these torque equations to work out accelerations, it is possible to work out the upper-body $\left(m_{1}\right)$ motion for a walking gait. The swinging foot $\left(m_{2}\right)$ is assumed to have a sinusoidal motion where $\mathrm{T}$ is the time to complete one whole cycle of the gait:

$$
x_{2, t}=-2 x_{s} \cdot \cos \left(\frac{2 \pi}{T} t\right)
$$

The equation for motion for $m_{1}$ is then: 


$$
x_{1, t}=C_{1} \cosh \left(a_{1} t\right)+C_{2} \sinh \left(a_{1} t\right)+C_{3} \cos (\omega t)
$$

Where:

$$
\begin{gathered}
C_{1}=x_{1,0}-C_{3} \quad C_{2}=\frac{v_{1,0}}{a_{1}} \\
C_{3}=\frac{m_{2} g 2 x_{s}}{m_{1} z_{1}\left(\alpha_{1}^{2}+\omega\right)} \quad \omega=\frac{2 \pi}{T} \\
\alpha_{1}=\sqrt{\frac{g}{z_{1}}}
\end{gathered}
$$

The 3-MLIPM appears to provide a much more accurate model of the motion of a robot while walking compared to the IPM, showing that the swinging leg has the effect of pulling on the central mass $\left(m_{1}\right)$ of the body. From equation (3) we are able to work out the position of the main body mass with an initial velocity and initial position. So far we have used this to generate a walking gait.

\section{$3 \quad$ Identifying a De-Stabilising Impact}

To identify shorty after impact whether a corrective action is needed, a model of the stability of the robot needs to be created. Here, the 3-MLIPM will be expanded upon. Unlike equation (3) we are not looking at the leg already in motion, as during a normal walking gait. Here, all masses are initially static. The masses $m_{1}$ for the mass of the main body of the robot, and $m_{2}$ for the mass of the leg that is not being used as the pivot will be considered. These are at positions $\left(x_{1}, z_{1}\right)$ and $\left(x_{2}, 0\right)$ respectively (figure 1$)$. Using equation (1) we get the formula:

$$
m_{1} \ddot{x}_{1} z_{1}=m_{1} x_{1} g+m_{2} x_{2} g \quad \rightarrow \quad \ddot{x}_{1}=x_{1} \frac{g}{z_{1}}+\frac{m_{2} x_{2} g}{m_{1} z_{1}}
$$

For simplicity the equation is re-written as:

$$
\ddot{x}_{1}=\alpha_{1}^{2} \cdot x_{1}-\alpha_{2}
$$

Where $\alpha_{1}$ remains the same as in equation (4) and:

$$
\alpha_{2}=\frac{-m_{2} x_{2} g}{m_{1} z_{1}}
$$

As an estimate based on equation (3) the formula for motion should be able to be represented as an equation in the form:

$$
x_{1, t}=D_{1} \cosh \left(\alpha_{1} t\right)+D_{2} \sinh \left(\alpha_{1} t\right)+D_{3}
$$

From this the first and second derivatives are then calculated: 


$$
\begin{aligned}
\dot{x}_{1, t} & =D_{1} \alpha_{1} \sinh \left(\alpha_{1} t\right)+D_{2} \alpha_{1} \cosh \left(\alpha_{1} t\right) \\
\ddot{x}_{1, t} & =D_{1} \alpha_{1}^{2} \cosh \left(\alpha_{1} t\right)+D_{2} \alpha_{1}^{2} \sinh \left(\alpha_{1} t\right) \\
& =\alpha_{1}^{2} \cdot x_{1}-D_{3} \alpha_{1}^{2}
\end{aligned}
$$

For (8) to be correct, (11) should be equal to (6). This is the case if:

$$
D_{3} \alpha_{1}^{2}=\alpha_{2}
$$

Therefore:

$$
D_{3}=\frac{\alpha_{2}}{\alpha_{1}^{2}}
$$

To workout $D_{1}$ and $D_{2}$ we take the case where time is set to zero $(t=0)$. Using equation (8):

$$
D_{1}=x_{1,0}-D_{3}
$$

Using equation (9):

$$
\dot{x}_{1,0}=v_{0} \quad D_{2}=\frac{v_{0}}{\alpha_{1}}
$$

This means that it is possible to predict the motion of the robot based on an initial position $\left(x_{1,0}\right)$ and an initial velocity $\left(v_{0}\right)$ resulting from the impact.
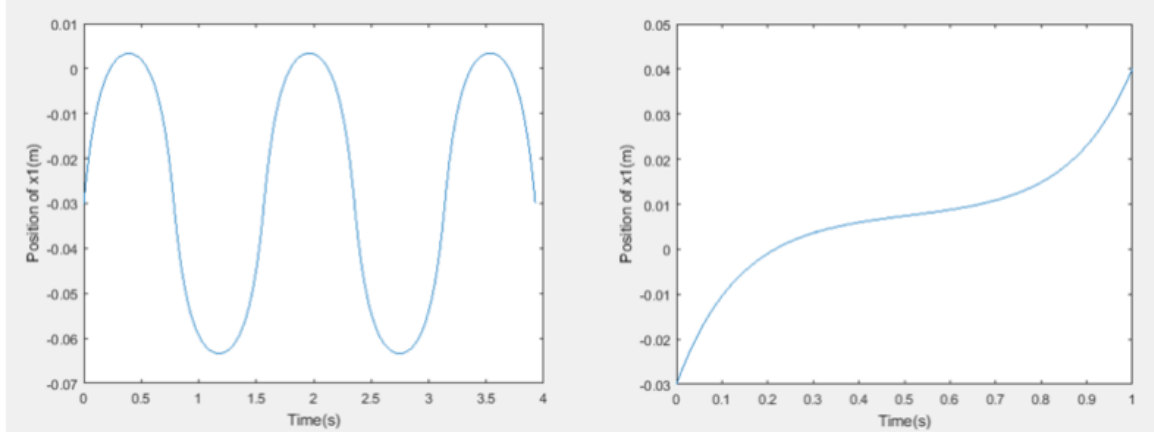

Fig. 2. Graphs showing model of position of $x_{1}$. On the left, the motion of $x_{1}$ in a stable system. On the right the motion of $x_{1}$ in an unstable system.

If the initial velocity is low enough then $x_{1}$ will oscillate between a front and back excursion amplitude, eventually coming to a stop due to friction. For an amplitude beyond a given tipping point the position of $x_{1}$ will continue to 
accelerate. The model assumes that the height stays constant which allows the speed to increase infinitely. However, in reality, the legs will no longer be able to maintain this height and the robot will fall over. The tipping point of the robot is slightly beyond the pivot leg. This position can be worked out by calculating when acceleration is zero by using equation (10), which resolves to the value of $D_{3}$.

So for the robot to remain stable, the velocity of the position of $x_{1}$ must reach zero. By using equation (9) the robot can be determined to be stable if the following equation is true at some value of time:

$$
\frac{v_{0}}{a_{1}\left(D_{3}-x_{1,0}\right)}=\tanh \left(\alpha_{1} t\right)
$$

As the function tanh has two horizontal asymptotes at $y=-1$ and $y=1$, and time can only have a positive value the following constraint can be made if equation (16) is to evaluate to true at some point in time, thereby showing that the system is stable:

$$
0 \leq \frac{v_{0}}{a_{1}\left(D_{3}-x_{1,0}\right)}<1
$$

This constraint means that by looking at the initial velocity, and the initial position it is possible to immediately decide if the robot will become unstable and fall, thereby being able to start the corrective action as soon as possible.

\section{Calculating a Stabilizing Response}

Once a de-stabilising event has been detected, the robot controller needs to react quickly and efficiently. To do this we need to calculate the new position for the robot to move one of its feet to stabilise itself. To calculate this, the motion needs to be re-calculated considering the fact that the robot now has a swinging foot with a non-zero mass. It is assumed that the swinging foot has a sinusoidal motion, similar to equation (2). However, unlike the previous equation the new position of $x_{2}$ is not a fixed distance ahead of the previous pivot point but needs to be determined based on the impact. This position will be called $x_{2, t^{\prime}}$, where $t^{\prime}$ is the time where the swinging foot touches the ground, becoming the new pivot foot:

$$
x_{2, t^{\prime}}=x_{2,0}+\frac{1}{2}\left(x_{2, t^{\prime}}-x_{2,0}\right) \cdot(1-\cos (\omega t))
$$

The new equations for the motion of the robot based on the torque equation (1) and motion of the foot becomes:

$$
\begin{aligned}
\ddot{x}_{1} & =x_{1} \frac{g}{z_{1}}+\frac{m_{2} g}{m_{1} z_{1}}\left(x_{2,0}+\frac{1}{2}\left(x_{2, t^{\prime}}-x_{2,0}\right) \cdot(1-\cos (\omega t))\right) \\
& =\alpha_{1}^{2} x_{1}+\alpha_{3} x_{2,0}+\frac{\alpha_{3} \alpha_{4}}{2}-\frac{\alpha_{3} \alpha_{4}}{2} \cos (\omega t)
\end{aligned}
$$


Where $\alpha_{1}$ remains the same as in equation (4), $\omega$ represents the angular velocity, and:

$$
\alpha_{3}=\frac{m_{2} g}{m_{1} z_{1}} \quad \alpha_{4}=x_{2, t^{\prime}}-x_{2,0}
$$

Again the model for the position of the robot is estimated based on equation (3):

$$
x_{1, t}=E_{1} \cosh \left(\alpha_{1} t\right)+E_{2} \sinh \left(\alpha_{1} t\right)+E_{3} \cos (\omega t)+E_{4}
$$

The first and second derivatives are calculated:

$$
\begin{aligned}
\dot{x}_{1, t} & =E_{1} \alpha_{1} \sinh \left(\alpha_{1} t\right)+E_{2} \alpha_{1} \cosh \left(\alpha_{1} t\right)-E_{3} \omega \sin (\omega t) \\
\ddot{x}_{1, t} & =E_{1} \alpha_{1}^{2} \cosh \left(\alpha_{1} t\right)+E_{2} \alpha_{1}^{2} \sinh \left(\alpha_{1} t\right)-E_{3} \omega^{2} \cos (\omega t) \\
& =\alpha_{1}^{2} x_{1, t}-\alpha_{1}^{2} E_{4}-\left(\alpha_{1}^{2} E_{3}+E_{3} \omega^{2}\right) \cdot \cos (\omega t)
\end{aligned}
$$

For equation (22) to be correct, equation (25) should be equal to equation (20). This is the case if:

$$
E_{3}=\frac{\alpha_{3} \alpha_{4}}{2\left(\alpha_{1}^{2}+\omega^{2}\right)} \quad E_{4}=\frac{2 \alpha_{3} x_{2,0}+\alpha_{3} \alpha_{4}}{-2 \alpha_{1}^{2}}
$$

$E_{1}$ and $E_{2}$ are worked out by looking at the case where time is set to zero $(t=0)$. Using equation (22) for $E_{1}$ and (23) for $E_{2}$ :

$$
E_{1}=x_{1,0}-E_{3}-E_{4} \quad E_{2}=\frac{v_{0}}{\alpha_{1}}
$$

The position needs to be calculated based on the swinging foot having reached its front position $\left(t^{\prime}\right)$. This occurs when:

$$
\cos \left(\omega t^{\prime}\right)=-1
$$

The position and velocity is now calculated based on this:

$$
\begin{aligned}
x_{1, t^{\prime}} & =E_{1} \cosh \left(\alpha_{1} t^{\prime}\right)+E_{2} \sinh \left(a_{1} t^{\prime}\right)-E_{3}+E_{4} \\
v_{t^{\prime}} & =E_{1} \alpha_{1} \sinh \left(\alpha_{1} t^{\prime}\right)+E_{2} \alpha_{1} \cosh \left(\alpha_{1} t^{\prime}\right)
\end{aligned}
$$

By adjusting equation (17) to look at the new position of the robot an analysis can be made for the position and velocity to see if this will stabilise the robot:

$$
0 \leq \frac{v_{t^{\prime}}}{\alpha_{1}\left(\frac{\alpha_{3} x_{2, t^{\prime}}}{\alpha_{1}^{2}}-x_{1,0^{\prime}}\right)}<1
$$


As the position used in these equations is relative to the position of the pivot foot:

$$
x_{1,0^{\prime}}=x_{1, t^{\prime}}-x_{2, t^{\prime}}
$$

Using these equations and expanding out all the terms that contain $x_{2, t^{\prime}}$ the minimum movement required to stabilise the robot can be found:

$$
x_{2, t^{\prime}}>\frac{\alpha_{7}\left(E_{2}+x_{1,0}\right)+\alpha_{3} x_{2,0}\left(\frac{1+\alpha_{7}}{\alpha_{5}}+\frac{1-\alpha_{7}}{\alpha_{6}}\right)}{1+\alpha_{3}\left(\frac{1}{\alpha_{1}^{2}}+\frac{1+\alpha_{7}}{\alpha_{5}}+\frac{\alpha_{7}-1}{\alpha_{6}}\right)}
$$

Where:

$$
\begin{array}{r}
\alpha_{5}=2\left(\alpha_{1}^{2}+\omega^{2}\right) \quad \alpha_{6}=-2 \alpha_{1}^{2} \\
\alpha_{7}=\cosh \left(\alpha_{1 t^{\prime}}\right)+\sinh \left(\alpha_{1 t^{\prime}}\right)
\end{array}
$$

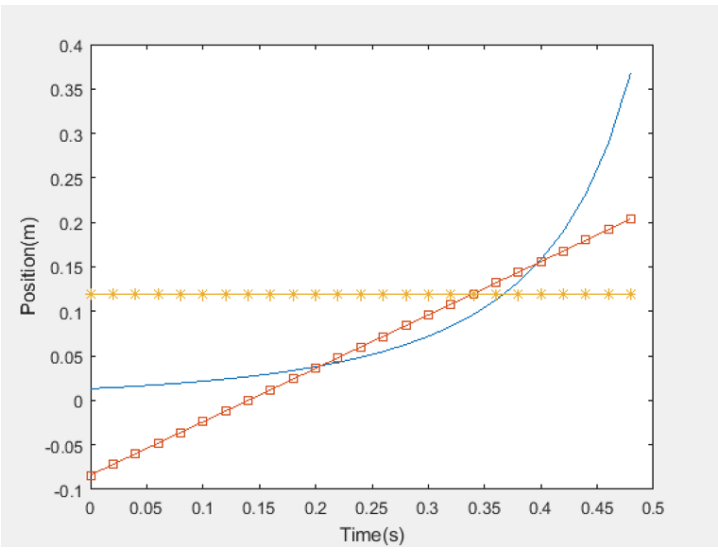

Fig. 3. A graph showing an example of the balance region for a robot. The line without a marker shows the minimum step required defined by equation (33). The square marked line shows the maximum position reachable by the foot based on it having a simple linear speed, in reality this would need to be calculated based on the configuration of the leg and servos. The star marked line shows an example of the maximum position the foot can reach.

With a calculation for the minimum step, and knowledge of the maximum speed and reach of the leg, a region of coordinates in time and space can be shown. Figure 3 shows an example of this region below the marked lines representing the position the leg can reach and the speed it can reach the position at, and above the unmarked line showing the minimum step required. This region then represents the possible movements of the leg that will balance the robot in a single step. 


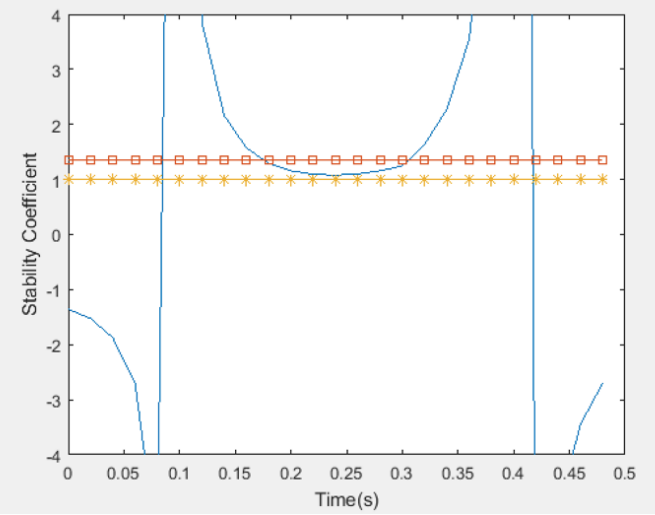

Fig. 4. A graph showing the stability coefficient of the robot. The square marked line represents the initial stability calculated using equation (17). The line without a marker is the stability coefficient assuming the robot moves the leg at an assumed maximum possible speed using equation (30). The star marked line shows the stability that needs to be reached. Note the lines at 0.08 and 0.42 are caused by asymptotes and do not reflect true values.

In many cases it is likely that a single step will not be enough to stabilise the robot. In this case the robot needs to take a sequence of corrective steps. Each step should have the effect of reducing the overall speed that has been created by the impact. This can be done by analysing the stability coefficient of a potential step and seeing if this can be made smaller than the previous stability coefficient, while remaining over 0 .
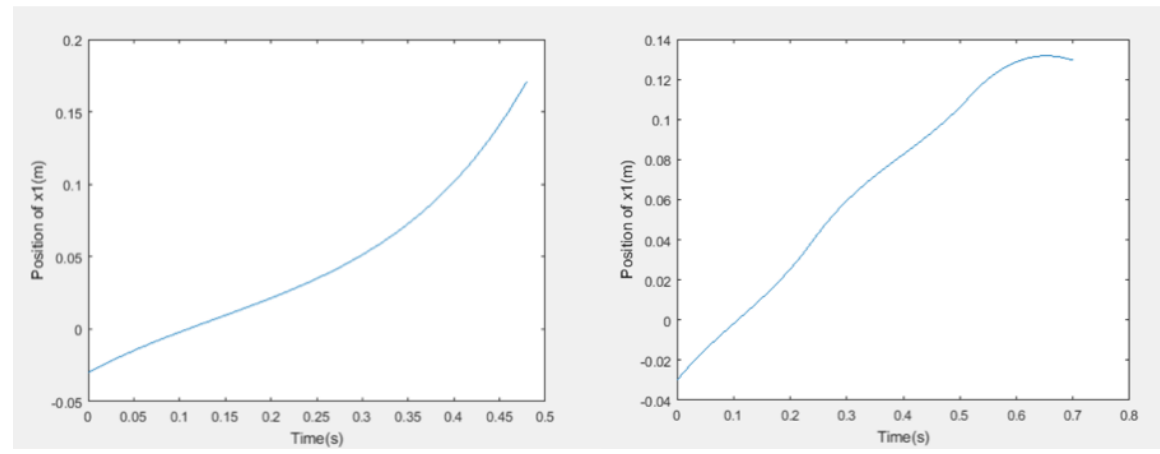

Fig. 5. Graphs showing a simulated impact and reaction of a robot. On the left the position if the robot takes no action, showing a fall. On the right the robot takes two steps. Step 1 is completed at 0.24 seconds and step 2 is completed at 0.52 seconds. 
If this reduction of the stability coefficient continues then the robot reaches a state where one more step will lead to a balanced state of the robot as shown in figure 5. This shows that it is possible to balance the robot if an initial speed and position of the main mass of the robot can be calculated compared to the position of the leg.

\section{Conclusion}

This model shows that knowing the position of the robot and the velocity created by the impact, an immediate decision can be made as to whether a reaction is necessary, and the stepping sequence that will correct the instability.

To actually implement this in a robot there are two main challenges that need to be resolved. The first is to measure the velocity resulting from the impact. Using an accelerometer and integrating the values to get a measurement for speed seems the most likely solution but the accelerometer needs to be able to sample at a high enough frequency, and drift accounted for.

The second challenge that needs to be overcome is the lateral motion of the robot required to lift the leg. Bugmann's [2] paper on the 3-MLIPM describes the lateral motion during a walking gait, where the lateral motion is at maximum velocity when both feet are on the ground, and acceleration is minimal. However, in the case of a robot suddenly reacting to an external impact the opposite is true, the robot has no velocity, and needs maximum acceleration to account for this.

\section{References}

1. Buczek, F.L., Cooney, K.M., Walker, M.R., Rainbow, M.J., Concha, M.C., Sanders, J.O.: Performance of an inverted pendulum model directly applied to normal human gait. Clinical Biomechanics 21(3), 288-296 (2006)

2. Bugmann, G.: Joint torques and velocities in a 3 -mass linear inverted pendulum model of bipedal gait. In: Conference Towards Autonomous Robotic Systems. pp. 82-93. Springer (2015)

3. Kajita, S., Kanehiro, F., Kaneko, K., Yokoi, K., Hirukawa, H.: The 3d linear inverted pendulum mode: A simple modeling for a biped walking pattern generation. In: Intelligent Robots and Systems, 2001. Proceedings. 2001 IEEE/RSJ International Conference on. vol. 1, pp. 239-246. IEEE (2001)

4. Yunping, L., Lipeng, W., Ping, M., Kai, H.: Stability analysis of bipedal robots using the concept of lyapunov exponents. Mathematical Problems in Engineering 2013 (2013) 